\title{
REDUCTION IN INFECTION WITH PLASMODIUM FALCIPARUM ONE YEAR AFTER THE INTRODUCTION OF MALARIA CONTROL INTERVENTIONS ON BIOKO ISLAND, EQUATORIAL GUINEA
}

\author{
IMMO KLEINSCHMIDT,* BRIAN SHARP, LUIS E. BENAVENTE, CHRIS SCHWABE, MIGUEL TORREZ, \\ JAIME KUKLINSKI, NATASHIA MORRIS, JAISHREE RAMAN, AND JOSEPH CARTER \\ Malaria Research Programme, Medical Research Council of South Africa, Durban, South Africa; Medical Care Development \\ International, Silver Spring, Maryland; One World Development Group, Gainesville, Florida
}

\begin{abstract}
The Bioko Island Malaria Control Project was initiated in 2003 to substantially reduce malaria on the island of Bioko in Equatorial Guinea. The intervention consisted of generalized indoor residual spraying during the first year of the project. Case management and related measures were introduced during the second year. Two large household and parasitemia surveys of children 2 to $<15$ years of age were carried out in 2004 and 2005 , respectively, to assess the effect of the intervention after the first year. Patient records were collected retrospectively from one district hospital and analyzed for a comparison of pre-intervention and post-intervention periods. Overall mean prevalence of $P$. falciparum infection reduced from 46\% (95\% confidence interval $[\mathrm{CI}]=40-51 \%)$ at baseline in 2004 to $31 \%(95 \%$ $\mathrm{CI}=24-40 \%)$ in $2005(P<0.001)$. When the 12-month pre-intervention period was compared with the 12-month post-intervention period, there was a modest but statistically significant reduction in the number of malaria cases among hospital patients.
\end{abstract}

\section{INTRODUCTION}

The Bioko Island Malaria Control Project (BIMCP) was initiated in 2003 to substantially reduce malaria on the island of Bioko, Equatorial Guinea. The project is funded by a consortium led by Marathon Oil Company (Houston, TX) in collaboration with the government of Equatorial Guinea, and implemented by a number of organizations.

Bioko has a population of approximately 250,000 inhabitants, of whom more than half live in and around the capital of Malabo. The discovery of offshore oil and gas in the 1990s has led to a rapid transformation of the economy. Malaria is endemic on Bioko, with very high transmission intensities; the predominant malaria vectors on the island are Anopheles gambiae s.s. and An. funestus. ${ }^{1}$

The BIMCP intervention is a two-pronged strategy consisting of vector control through routine indoor residual spraying (IRS) of all domiciliary structures, and an extensive program of case management and intermittent preventive treatment (IPT) for pregnant women. The two arms of the intervention were staggered with the first round of IRS beginning in March 2004, and case management and IPT starting in February 2005. The effect of the project is being assessed through several evaluation tools. These are annual parasitemia and anemia household surveys, daily vector surveillance through a system of window traps whose contents are collected daily and analyzed, introduction of a comprehensive malaria information system recording all malaria-related illness episodes, an assessment of all cause mortality for children less than five years of age through household surveys of women at baseline and five years later, and an economic assessment of the effect of malaria at baseline and five years later through a household survey measuring the direct and indirect cost-of-illness and the demand for care. In addition, patient registers from one hospital were collected retrospectively, entered into a database, and pre-intervention and post intervention periods were compared. This report describes the results of the base-

\footnotetext{
* Address correspondence to Immo Kleinschmidt, Medical Research Council, 491 Ridge Road, Overport, Durban 4091, South Africa. Email: immo.kleinschmidt@mrc.ac.za
}

line and first annual follow-up parasitemia surveys, and the comparison of pre-intervention and post intervention patient registers.

\section{MATERIALS AND METHODS}

The first round of IRS to cover all structures (rooms) on the island was carried out between March 2004 and August 2004. Spray manuals were developed and sprayers were trained in all aspects of house-spraying including safety precautions, and obtaining informed consent from householders. Spray operations were similar to those developed in the Lubombo Spatial Development Initiative in Mozambique, Swaziland, and South Africa. ${ }^{2}$ Progress and performance of spray operations were continually monitored with the help of a computerized spray management system that was developed for this purpose. ${ }^{3}$ A total of 97,000 structures were sprayed with synthetic pyrethroids. Most structures were treated with Deltamethrin ${ }^{\mathrm{TM}}$ (Bayer, Leverkusen, Germany), and the remainder was sprayed with Fendona ${ }^{\circledR}$ (BASF Corporation, Ludwigshafen, Germany).

Case management and IPT were started in February 2005 in all government health facilities. This consisted of strengthening of diagnostic facilities including routine definitive diagnosis, introduction of oral artesunate in combination with sulfadoxine-pyrimethamine as first-line treatment, training of doctors and nurses in case management and IPT, and introduction of a pharmaceutical logistics and supervisory system. Anti-malarial treatment to children less than 15 years of age is provided free of charge. Each of these transmission reduction interventions are reinforced through a concerted information, education, and communications (IEC) initiative, a comprehensive monitoring and evaluation protocol, and a detailed integration strategy to ensure sustainability through capacity building.

The effect of the first round of IRS was assessed by comparing parasitemia data from household surveys carried out before the start of spraying in February-March 2004 and postspraying in February-April 2005 and by comparing hospital registers coinciding with 12 -month pre-intervention and 12 - 
month post-intervention periods. The combined effect of IRS and the case management program will be assessed by subsequent surveys.

A system of 18 sentinel sites was set up for monitoring and evaluation by means of annual household surveys, and daily monitoring of mosquito catches from window traps installed at designated houses at these sites (Figure 1). The baseline survey at two sites was carried out in June 2004; a third site was not included in the 2004 survey. This resulted in 15 sentinel sites at which results from the two surveys could be directly compared. Using geographic information systems, we defined five surveillance areas using Thiessen/Voronoi polygons ${ }^{4}$ based loosely on the four administrative districts of Malabo, Luba, Riaba, and Baney, with Malabo partitioned into a metro and rural component.

A survey sample size of 110 children per site was calculated to ensure $80 \%$ power for detecting a true change in prevalence of infection of $20 \%$ at each sentinel site, with a $5 \%$ type 1 error rate (significance level). Although it could reasonably be assumed that there would be on average two children between 2 and 15 years of age in each household, it was decided to set a target of 80 households per sentinel site for each survey round to compensate for the fact that some school children may not be available during visits, and to allow for a design effect that would arise from within-household correlation of responses at each sentinel site.

In each survey round an advance field-team visited each site. Houses in urban sites were identified from randomly pre-selected coordinates using global positioning systems and satellite images. Houses in rural sites were selected by systematic sampling from hand-drawn maps. Householders were contacted to explain the purpose of the survey, obtain in-

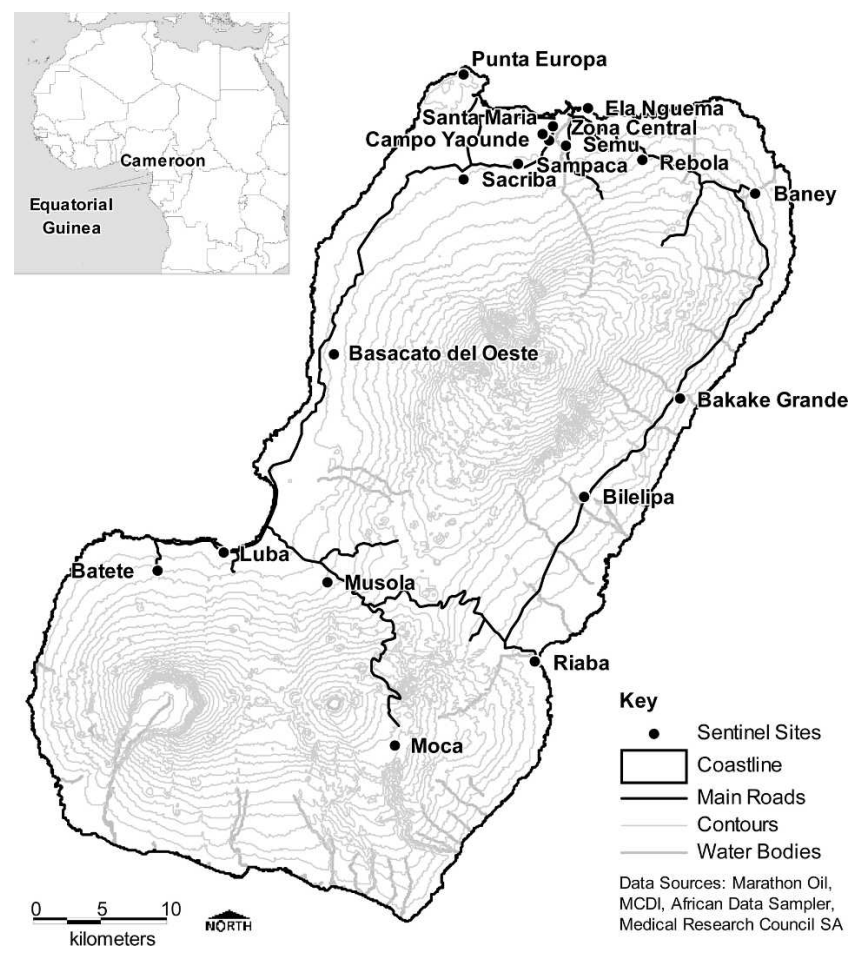

FIGURE 1. Location of sentinel sites used to monitor the Bioko Island Malaria Control Project intervention. MCDI $=$ Medical Care Development International; SA = South Africa. formed consent, and arrange a time for a fieldworker from the survey team to call on the house. Survey teams consisting of trained nurses visited selected houses and administered a questionnaire to caregivers; finger prick blood samples were taken from all attending children between the ages of 2 and $<15$ years to assess malarial parasitemia with ICT ${ }^{\mathrm{TM}}$ malaria rapid tests (ICT Diagnostics, Sydney, New South Wales, Australia) and measure hemoglobin levels with photometers (HemoCue Inc., Lake Forest, CA). The body temperature of each child was measured using a digital thermometer. Children who tested positive for parasitemia, had a hemoglobin level $<7.0 \mathrm{~g} / \mathrm{dL}$, or had fever (temperature $>36.99^{\circ} \mathrm{C}$ ) where offered treatment at a local field clinic set up for this purpose.

The survey instrument covered questions relating to whether the house was sprayed during the previous year, attitudes towards IRS, awareness of IEC messages, and illness history of household members during the preceding four-week period. In the 2005 survey pregnant women were also assessed for malarial parasitemia, hemoglobin levels, and body temperature, and were asked whether they took iron supplementation or preventive treatment against malaria.

Completed questionnaires were checked daily by the survey supervisor so that corrections could be made if necessary by revisiting households. Checked questionnaires were entered by locally trained data entry personnel into a Microsoft (Redmond, WA) Access ${ }^{\mathrm{TM}}$ database. Entered records were verified by reading them back to a second transcriber who compared them to the relevant questionnaire.

After transcription, data were cleaned and checked for inconsistencies. Household records were converted to individual child records. Statistical analysis took account of the survey design using the correction of Rao and $\mathrm{Scott}^{5}$ as implemented in STATA software, ${ }^{6}$ thereby correcting standard errors for multistage sampling. ${ }^{7}$ All analysis calculating results for all sites or a subset of sites was carried out assuming the sentinel site to be the primary sampling unit (PSU); all calculations that attempted to produce site-specific results assumed the household to be the PSU. All analysis comparing 2005 results with those of 2004 was restricted to the 15 sites that were surveyed in both years from February to April, and to children 2 to $<15$ years of age, unless stated otherwise.

Prevalence was calculated and plotted for individual years of age by survey round (2004 and 2005) for all children in the comparison sites. Confidence intervals (CIs) for each age group were calculated from the survey design adjusted standard errors.

A logistic regression model for Plasmodium falciparum infection including terms for post-intervention (2005 survey), age of child $<5$ years relative to $\geq 5$ years, and an interaction term for intervention and age $<5$ years was used to test whether there was significant heterogeneity in the intervention effect between younger and older children. A further logistic regression model was used to test whether the intervention effect differed between the five surveillance areas.

Validation of ICT rapid test results was carried out on a sample of 153 children. In addition to conducting the standard ICT malaria rapid test, thick and thin blood smears from each child were independently examined by three microscopists, and dried finger prick blood samples blotted on filter paper were assessed for the presence of $P$. falciparum DNA using a nested polymerase chain reaction (PCR) at The Medical Research Council laboratories in Durban, South Africa. ${ }^{8,9}$ 
Patient registers covering the period from March 1, 2003 to February 28, 2005 from Luba district hospital were retrospectively transcribed into a Microsoft Access database that did not include patient identifiers and names. Luba was chosen after an inspection of registers at all district hospitals because it had the most comprehensive patient registers. All pediatric outpatient records were entered $(\mathrm{n}=2,843)$, and all inpatient records from the adult register were captured $(n=1,309)$. Inpatient pediatric cases had not been recorded in a structured manner in the registers and were therefore not entered. According to the hospital superintendent at Luba, all inpatient malaria diagnoses were confirmed by blood smears examined in the hospital's laboratory. Outpatient malaria diagnoses were based in some instances on a laboratory result, and in others a clinical diagnosis was made. The outpatient register did not indicate the type of diagnosis.

The Ministry of Health and Social Welfare of the Government of Equatorial Guinea gave ethical clearance for the BIMCP and for the surveys.

\section{RESULTS}

Parasitemia. In the baseline survey, 2,236 children from 951 households at 15 sentinel sites were surveyed. In the 2005 follow-up survey, 2,645 children 2 to $<15$ years of age from 1,193 households at the same sentinel sites were assessed for parasitemia. Data from three sites were not included in analysis comparing 2005 with baseline because they were either not surveyed at baseline or they were surveyed at a different time of year. Apart from the age stratified analysis, all other analysis was restricted to children $\geq 2$ years of age because this was a selection criterion in the baseline survey.

Overall prevalence of infection with $P$. falciparum across all 15 sites surveyed in February-March 2004 was 46\% (95\% $\mathrm{CI}=40-51 \%)$. The mean prevalence in the Malabo sites was $39.1 \%$ compared with $48.5 \%$ for mainly rural sites outside Malabo $(P=0.13)$. Overall prevalence in 2005 for the same
15 sites was significantly lower at $31 \%(95 \% \mathrm{CI}=24-40 \%$, $P=0.0009)$. In 2005, prevalence in Malabo was $25.5 \%(95 \%$ $\mathrm{CI}=17.7-35.2 \%)$ compared with $33.6 \%$ (95\% CI $=24.2-$ $44.6 \%)$ at non-Malabo sites $(P=0.22)$. There was considerable between site variation in prevalence, particularly in 2005 (Table 1). The overall odds ratio (OR) for prevalence of infection comparing 2005 with 2004 was 0.53 (95\% CI $=0.39$ $0.73)$, with significant heterogeneity in the reductions between the five surveillance areas $(P=0.003)$ (Table 2 and Figure 2). The reductions were greatest in metropolitan Malabo, Luba, and Baney, with more modest reductions in rural Malabo and Riaba. The change in prevalence of infection was significant in three of the five surveillance areas.

Prevalence of infection varied by age, with age-specific prevalence increasing gradually up to an age of seven years before decreasing in children more than 10 years of age (Figure 3). Logistic regression showed that children less than five years of age generally had a significantly lower risk of infection compared with older children $(\mathrm{OR}=0.78,95 \% \mathrm{CI}=$ $0.66-0.92, P=0.006)$. For children less than five years of age, the reduction in prevalence of infection between 2004 and $2005(\mathrm{OR}=0.43,95 \% \mathrm{CI}=0.31-0.61)$ was greater than that for older children $(\mathrm{OR}=0.57,95 \% \mathrm{CI}=0.41-0.80)$, but this difference in intervention effects was only marginally significant $(P=0.06)$.

Children in large households ( $>10$ members) were at significantly higher risk for infection than children in smaller households (prevalence $=41 \%$ versus $29 \% ; P=0.020$ ). There was no evidence that children in households whose heads had less than five years of education were more likely to be infected than those whose household head had more schooling $(P=0.57)$.

Of the 220 pregnant women who were tested for malarial parasitemia in 2005, 18.2\% (95\% CI $=10.9-25.4 \%)$ were positive. Thirty-nine (19\%) of 202 pregnant women reported receiving IPT. There was no evidence of an association between IPT and infection with malarial parasites $(P=0.61)$.

The validation study showed that in comparison to PCR,

TABLE 1

Prevalence of infection with Plasmodium falciparum in children 2 to 15 years of age, by sentinel site, observed during household surveys in 2004 and 2005 on Bioko Island, Equatorial Guinea

\begin{tabular}{|c|c|c|c|c|c|}
\hline \multirow[b]{2}{*}{ Sentinel site } & \multicolumn{2}{|c|}{ February/March 2004} & \multicolumn{2}{|c|}{ February/March 2005} & \multirow[b]{2}{*}{$P$} \\
\hline & $\begin{array}{l}\text { Prevalence of } \\
\text { infection, \% } \\
\text { (n) }\end{array}$ & $\begin{array}{c}95 \% \\
\text { Confidence } \\
\text { interval }\end{array}$ & $\begin{array}{l}\text { Prevalence } \\
\text { of infection, } \\
\% \text { (n) }\end{array}$ & $\begin{array}{c}95 \% \\
\text { Confidence } \\
\text { interval }\end{array}$ & \\
\hline Campo Yaounde & $36(220)$ & $28-44$ & $15(232)$ & $10-21$ & $<0.001^{*}$ \\
\hline Ela Nguema & 39 (105) & $28-51$ & $30(235)$ & $23-39$ & 0.21 \\
\hline Semu & 54 (182) & $45-62$ & $35(231)$ & $28-43$ & $0.001 *$ \\
\hline Central & $28(170)$ & $19-38$ & $22(183)$ & $16-30$ & 0.34 \\
\hline $\begin{array}{l}\text { Basacato del } \\
\text { Oeste }\end{array}$ & $62(92)$ & $51-72$ & $44(134)$ & $35-53$ & $0.01 *$ \\
\hline Luba & 41 (195) & $32-51$ & 38 (168) & $30-47$ & 0.66 \\
\hline Batete & $51(162)$ & $42-61$ & $15(159)$ & $10-23$ & $<0.001 *$ \\
\hline Riaba & $58(84)$ & $48-68$ & 54 (186) & $46-62$ & 0.53 \\
\hline Sampaka & 40 (181) & $32-48$ & $36(162)$ & $28-46$ & 0.59 \\
\hline Sacriba & $60(131)$ & $49-71$ & $58(182)$ & $50-65$ & 0.70 \\
\hline Musola & 44 (144) & $33-55$ & 13 (158) & $7-21$ & $<0.001 *$ \\
\hline Bilelipa & 48 (94) & $38-58$ & $50(104)$ & $39-61$ & 0.78 \\
\hline Rebola & 46 (158) & $38-53$ & $21(175)$ & $16-28$ & $<0.001^{*}$ \\
\hline Bakake Grande & $55(182)$ & $47-63$ & $24(160)$ & $17-34$ & $<0.001 *$ \\
\hline Baney & 40 (136) & $32-50$ & $21(174)$ & $14-29$ & $0.0013 *$ \\
\hline All & $46(2236)$ & $40-51$ & $31(2645)$ & $24-40$ & $0.0009 *$ \\
\hline
\end{tabular}

* Change since 2004 was statistically significant. 
TABLE 2

Odds ratios of infection with Plasmodium falciparum for 2005 relative to 2004, in children 2 to 15 years of age obtained from household surveys conducted at 15 sentinel sites, by surveillance area on Bioko Island, Equatorial Guinea*

\begin{tabular}{lcccc}
\hline \multicolumn{1}{c}{ Area } & $\begin{array}{c}\text { Prevalence in } 2004 \\
(95 \% \mathrm{CI}), \%\end{array}$ & $\begin{array}{c}\text { Prevalence in 2005 } \\
(95 \% \mathrm{CI}), \%\end{array}$ & $\begin{array}{l}\text { Odds ratio } \\
(95 \% \mathrm{CI})\end{array}$ \\
\hline Metropolitan & & & & \\
$\quad$ Malabo & $39.1(28.7-50.6)$ & $25.5(17.7-35.2)$ & $0.53(0.38-0.74)$ & $0.02(0.59-1.13)$ \\
Rural Malabo & $51.5(37.5-65.3)$ & $46.5(35.6-57.8)$ & $0.34(0.12-0.96)$ & 0.21 \\
Luba & $45.1(39.5-50.9)$ & $22.0(10.9-39.5)$ & $0.62(0.28-1.39)$ & 0.04 \\
Riaba & $54.2(49.7-58.6)$ & $42.5(26.2-60.6)$ & $0.34(0.29-0.39)$ & 0.23 \\
Baney & $43.2(39.3-47.2)$ & $20.5(16.1-25.7)$ & $0.53(0.39-0.73)$ & $<0.001$ \\
All areas & $45.7(40.3-51.2)$ & $30.9(23.7-39.2)$ & 0.001 \\
\hline
\end{tabular}

$* \mathrm{CI}=$ confidence interval.

the ICT malaria rapid tests had a sensitivity of $80 \%(95 \%$ $\mathrm{CI}=70-87 \%)$ and a specificity of $92 \%(95 \% \mathrm{CI}=81-98 \%)$. Combining the results of the three independent microscopy readings into a single majority verdict yielded a sensitivity of $82 \%(95 \% \mathrm{CI}=73-89 \%)$ and a specificity of $88 \%(95 \%$ $\mathrm{CI}=76-96 \%)$ for the combined microscopy relative to PCR.

Of 1,280 households who at the time of the 2005 survey lived in the same house they occupied in February 2004, $86.4 \%(95 \% \mathrm{CI}=80.8-92.0 \%)$ confirmed that their house was sprayed during 2004. Of all those questioned $(\mathrm{n}=1,386)$, $95.2 \%(95 \%$ CI $=93.5-96.8 \%)$ said they wanted their house to be sprayed in 2005 .
Patient records. The Luba Hospital inpatient register showed that in the 12 months before the start of the intervention from March 1, 2003 to February 28, 2004, 351 (52\%) of 681 adult patients had been diagnosed with malaria as their final diagnosis, including cases in which malaria was stated concomitantly with other diagnoses. Over the following 12month period from March 1, 2004 to February 28, 2005, 309 $(45 \%)$ of 687 patients had been diagnosed as malaria cases $(P=0.015)$. Of the pediatric outpatients, $543(47 \%)$ of 1,150 were diagnosed as malaria cases for the March 2003 to February 2004 period compared to $503(29 \%)$ of 1,730 malaria cases during the subsequent year $(P<0.001)$.

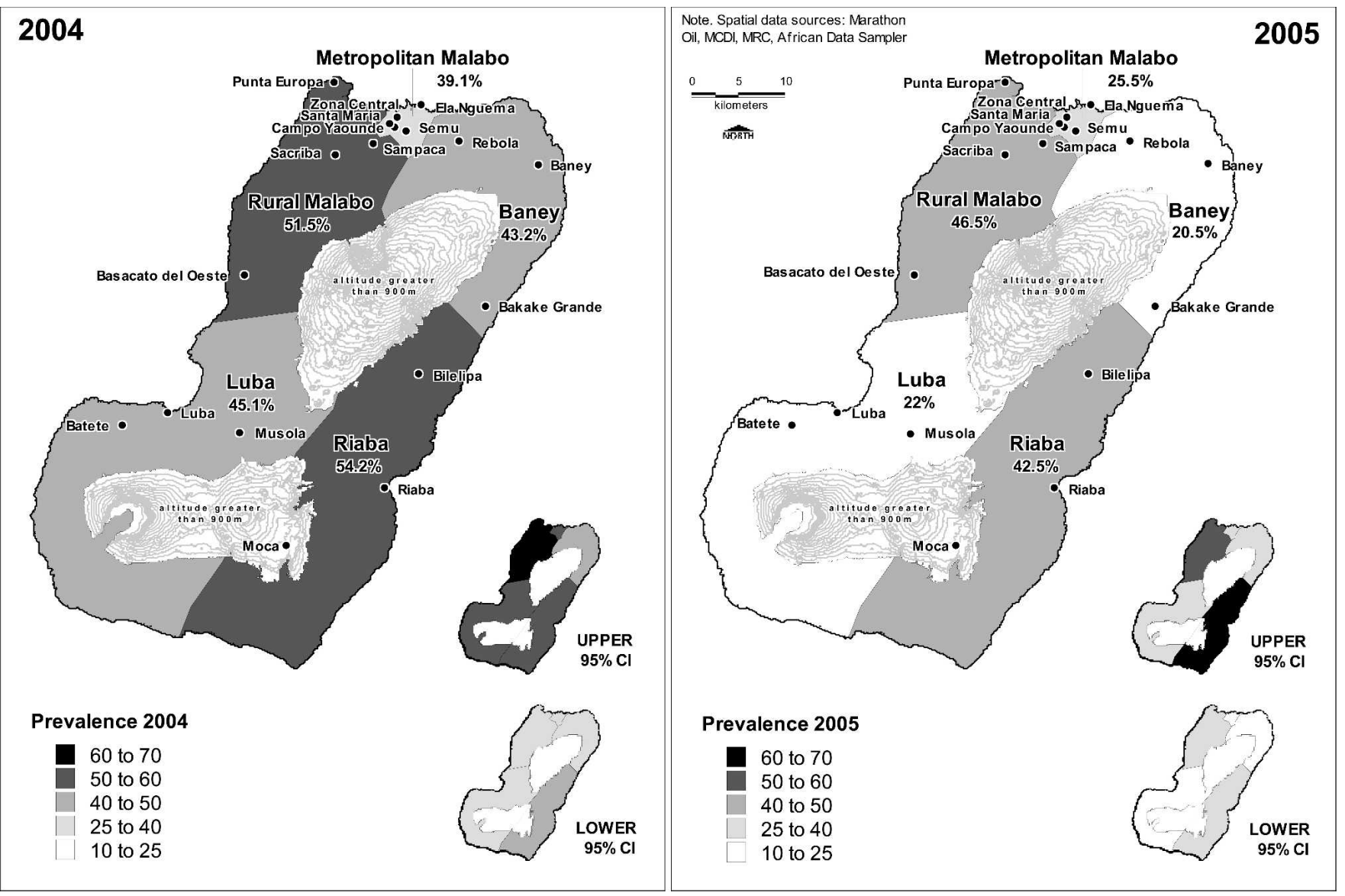

Figure 2. Estimated prevalence (\%) of Plasmodium falciparum in children 2 to $<15$ years of age, by surveillance area and survey year. Boundaries shown are artificial constructs created by generating Thiesen/Voronoi polygons around sentinel sites. CI $=$ confidence interval. 


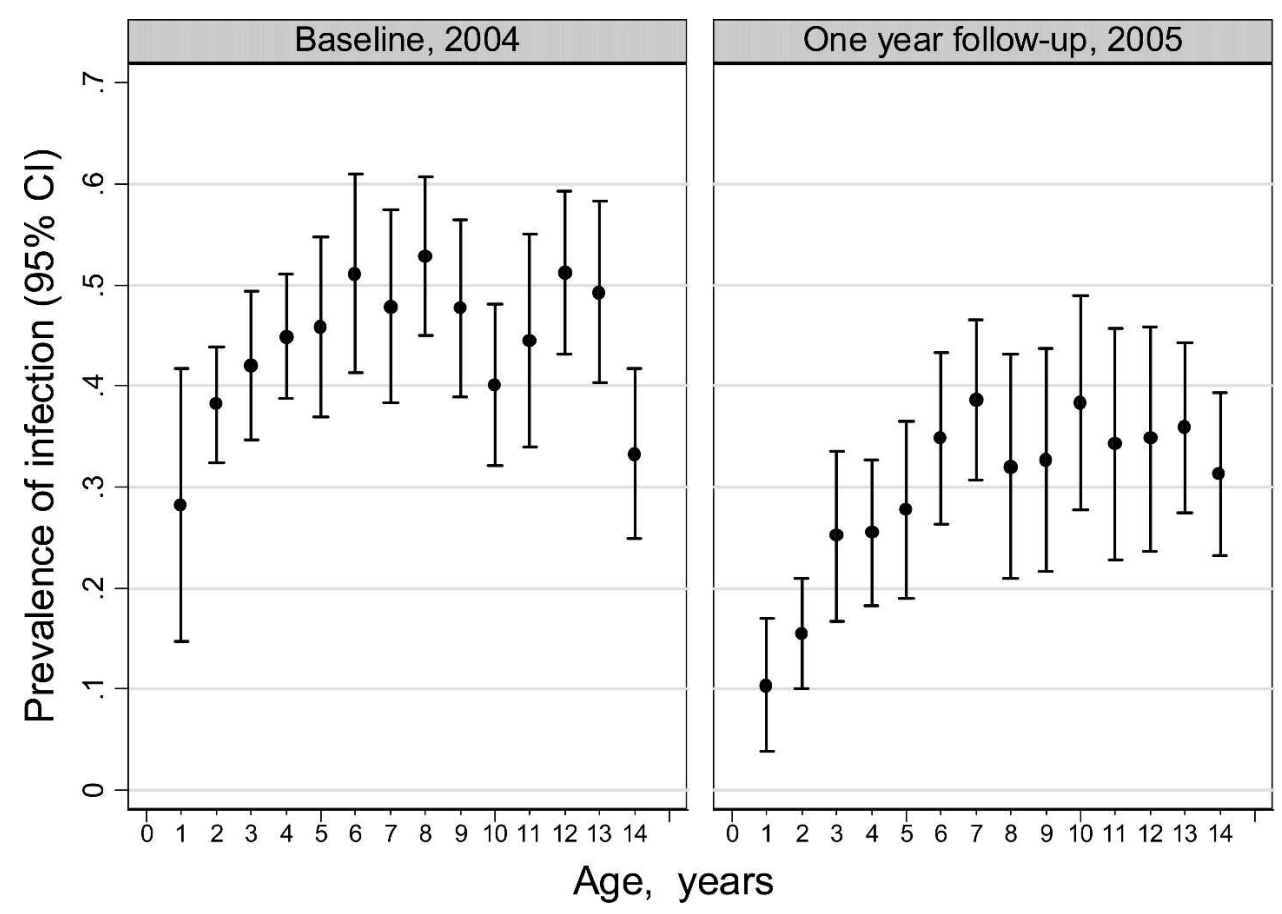

FIGURE 3. Plasmodium falciparum prevalence of infection by age. Error bars show $95 \%$ confidence intervals (CIs). One-year-old children were included in this analysis since the data were stratified by single year of age. At baseline, the sample of one-year-old children was disproportionately small, as reflected in the wide CI. Prevalence shown as a proportion.

\section{DISCUSSION}

Prevalence of infection with malaria parasites in children is widely used as a proxy measure for malaria transmission intensity (see for example MARA/ARMA ${ }^{10}$ ), despite the fact that the relationship between prevalence and transmission intensity, as measured by the entomologic inoculation rate (EIR), is not linear. ${ }^{11}$ Prevalence surveys in non-immune persons such as children give a good indication of the reservoir of infection in a population, and thus of transmission potential. Such surveys are relatively simple to conduct, particularly after the advent of rapid diagnostic test devices. ${ }^{12}$

The validation study of a sub-sample of 153 children in the survey showed that the rapid test used in both the 2004 and 2005 surveys performed well in relation to PCR, with sensitivity and specificity very similar to what was achieved by the combined results of three microscopists. This confirms the efficacy of the rapid test as an instrument for definitive diagnosis of $P$. falciparum malarial infection in the study area.

Parasite prevalence surveys have previously been conducted in Bioko, ${ }^{13,14}$ both among the general population and hospital patients. The results of these prevalence surveys varied widely. For example, Roche and others ${ }^{14}$ in 1991 observed an overall prevalence of $27 \%$ in a survey of more than 1,000 children from both high and low altitude villages. Surveys carried out in 1992 and 1993 showed prevalence rates ranging from $30 \%$ to $80 \%$, with significant inter-seasonal variation. ${ }^{12}$ The results of the baseline survey for this project fell within this range. A malaria distribution model based on more than 400 prevalence surveys conducted in west Africa predicted similar prevalence of infection for equatorial forest areas of west Africa. ${ }^{15}$

The results from the baseline survey showed strong spatial heterogeneity in infection prevalence despite the generally low altitude of most human population settlements on the island (Figure 2). The areas of highest prevalence appeared to be in the northwest (non-metro part of Malabo district) and southeast (Riaba district) areas, followed by the southwest (Luba district), northeast (Baney district), and metro areas of Malabo. These geographic differences persisted at a lower level at the time of the follow-up survey. The two areas with the highest levels of infection at baseline, non-metro Malabo and Riaba, had the weakest intervention effect. The smaller reduction in prevalence in areas of high baseline prevalence can be explained by the documented relationship between prevalence of infection in children (parasite ratios) and transmission intensity. ${ }^{11}$ At high transmission intensity, prevalence of infection is more or less insensitive to moderate changes in transmission intensity. Only once transmission intensity (as measured by the EIR) is reduced below a threshold value will there be corresponding reductions in prevalence. In areas of high prevalence in Bioko the reduction in transmission intensity achieved so far may be insufficient to translate into significant reductions in prevalence.

Given the high levels of transmission intensity in Bioko, one might have expected a peaking of prevalence at a younger age than the prevalence versus age graphs suggest ${ }^{16-18}$ (Figure 3). The significantly lower prevalence in children less than five years of age in our data indicates that these children may be less exposed to infective bites than their older counterparts for reasons that are unknown and may be behavioral, but that warrant further investigation. Our results show that children more than five years of age are highly vulnerable to malaria infection, challenging the widely held assumption that children less than five years of age are the group at highest risk. Malaria control strategies concentrating exclusively on younger children should be guided by evidence 
that they are actually the group most at risk. Estimates of prevalence of infection at individual years of age (Figure 3) at baseline and one year follow-up show that the generalized intervention by IRS has benefited children of all ages less than 15 in Bioko. The shape of the prevalence age curve for 2005 is similar to the one for the 2004 survey, albeit at lower levels of infection, with no apparent shift in age of peak prevalence. The logistic regression model indicated that after one year the benefit of the intervention for older children $(\mathrm{OR}=0.57,95 \% \mathrm{CI}=0.41-0.80)$ was very similar to that for younger children $(\mathrm{OR}=0.43,95 \% \mathrm{CI}=0.31-0.61)$ ( $P$ for interaction $=0.06)$. This is an important advantage of IRS since our surveys have shown that older children on Bioko are highly vulnerable to malaria infection.

The results of the 2005 survey demonstrate that children in large households are at significantly higher risk of infection than children in smaller households. Household size is negatively correlated with per capita incomes (Schwabe C, unpublished data), suggesting that children in the poorest households were at a higher risk for infection than children in the wealthier households because of the inability of these households to afford treatment and prevention measures. Conversely, schooling of less than five years for the head of the household showed no apparent association with infection.

It is estimated that in sub-Saharan Africa at least $75 \%$ of deaths attributable to malaria are in children less than five years of age. ${ }^{19,20}$ The relationship between malaria transmission intensity and mortality in Africa has been reviewed by Smith and others. ${ }^{21}$ Arudo and others ${ }^{22}$ showed that using verbal autopsy, more than half of post-neonatal under-five mortality in rural Kenya was malaria related. A significant reduction in malaria transmission should therefore have a considerable impact on child mortality in Bioko.

Historical patient registers from Luba district hospital were analyzed because these appeared to be reasonably comprehensive and well kept, and because Luba is the largest hospital outside Malabo. Since it is the only hospital in the district and due to its geographic isolation, patients from Luba district would generally have to attend this facility. There may be inconsistencies in the recording of malaria cases, particularly with outpatients, who were assessed using both clinical and definitive diagnoses. Inpatient registers are likely to be more reliable because a final diagnosis of malaria was based on a blood smear result. Both registers that were analyzed showed that malaria cases made up a large proportion of hospital cases, and therefore confirm the heavy burden that is placed on health services by the disease. Although caution is required in interpreting changes in recorded numbers of cases from such registers, we are encouraged that for both registers (pediatric outpatient and adult inpatient) the number of malaria cases recorded by the hospital has decreased significantly when comparing the 12-month period before spraying with the 12-month period after the intervention. This decrease in numbers of malaria cases seen at Luba Hospital further strengthens the evidence for a reduction in malaria transmission in Bioko since the advent of the BIMCP intervention.

The second annual parasitemia survey shows that there has been a substantial and significant overall reduction in prevalence of infection of approximately one third relative to the baseline prevalence of $46 \%$. All but one of the 15 sites showed a reduction in prevalence, but the magnitude of change varied between sites, and a concern is the geographic heterogeneity in reductions, with areas of highest prevalence at baseline achieving the smallest reductions. Since reported spray coverage was consistently high, the reason for the heterogeneity in the reductions of prevalence is not clear at this stage and requires further investigation. Previous studies in Bioko have reported EIRs as high as 281 and 788 infective bites per year by An. gambiae s.s. and An. funestus, respectively. ${ }^{1}$ In the light of these high transmission intensities at baseline, the reductions in prevalence of infection achieved after one year of IRS vector control are very promising. The combined effect of IRS and the remaining components of the intervention, namely effective diagnosis and treatment of malaria, IPT, and IEC, will only be apparent at the time of the third annual survey to be conducted in February 2006. A comprehensive malaria information system is currently being introduced to monitor changes in the incidence of symptomatic cases in the future.

Vector control programs using IRS have been successfully implemented in a number of countries in Africa, including Namibia, Botswana, Zambia, South Africa, Swaziland, and Mozambique. ${ }^{23,24}$ However, this is the first large-scale intervention of this kind in an equatorial setting in recent times, and the initial results are both encouraging and consistent with the southern African experience. We believe that its success is the result of a well-managed spraying operation that has achieved high coverage rates and a positive response from the general public, as shown by the almost universal desire to have houses re-sprayed in the second year. Continued reductions in prevalence of infection over the medium term are anticipated, and these will be consolidated by the successful implementation of the case management component of the intervention. Long-term success and sustainability of the combined intervention in controlling malaria transmission in Bioko will depend upon the successful transfer of the management and monitoring of the intervention to the Ministry of Health. The BIMCP has prepared a detailed integration strategy with the Ministry of Health that has mapped all project functions to relevant Ministry of Health units and evaluated the risks and influences that will either mitigate or foster effective transition and eventual sustainability. A detailed action plan specifies a range of capacity-building activities to include training, education, and mentoring. The strategy of initiating such a major malaria control project in an area of high transmission intensity as a private-public partnership is another example of a model that we believe can be successfully repeated elsewhere.

Received September 29, 2005. Accepted for publication January 6, 2006.

Acknowledgments: We thank Drs. Adel Chaouch, Brian Linder, and Susan Rynard (Marathon Oil Company) for their committed support during all stages of this project, the late Juanita Nzang (Ministry of Health and Social Welfare, Equatorial Guinea) for her support and insight into the importance of this project, and Roger Holeywell (Marathon Oil Company) for the contour map of Bioko Island. The American Society of Tropical Medicine and Hygiene (ASTMH) assisted with publication expenses.

Financial support: The BIMCP is wholly funded by a consortium led by the Marathon Oil Company.

Authors' addresses: Immo Kleinschmidt, Brian Sharp, Natashia Morris, and Jaishree Raman, Medical Research Council, 491 Ridge Road, Overport, Durban 4091, South Africa, Telephone: 27-31-203- 4806, 
Fax: 27-31-203-4704, E-mails: immo.kleinschmidt@mrc.ac.za, sharpb@mrc.ac.za,n.morris@mrc.ac.za, and j.raman@mrc.ac.za. Luis E. Benavente, Christopher Schwabe, Miguel Torrez, and Joseph Carter, Medical Care Development International, 8401 Colesville Road, Suite 425, Silver Spring MD 20910, Telephone: 301-562-1920, Fax: 301-562-1921, E-mails: lbenavente@mcd.org, chris@schwabe.us, mitorrez@mixmail.com, and jkcvermont@aol.com. Jaime Kuklinski, One World Development Group, 5745 SW 75 Street, \#219, Gainesville, FL 32608, Telephone and Fax: 352-262-6485, E-mail: jaime@ owdg.org.

\section{REFERENCES}

1. Cano J, Berzosa PJ, Roche J, Rubio JM, Moyano E, GuerraNeira A, Brochero H, Mico M, Edu M, Benito A, 2004. Malaria vectors in the Bioko Island (Equatorial Guinea): estimation of vector dynamics and transmission intensities. $J$ Med Entomol 41: 158-161.

2. Conteh L, Sharp BL, Streat E, Barreto A, Konar S, 2004. The cost and cost-effectiveness of malaria vector control by residual insecticide house-spraying in southern Mozambique: a rural and urban analysis. Trop Med Int Health 9: 125-132.

3. Booman M, Sharp BL, Martin CL, Manjate B, La Grange K, Durrheim DN, 2003. Enhancing malaria control using a computerized management system in Southern Africa. Malaria J 2: $1-5$.

4. MapInfo Professional version 7.5 supplement, 2003. Troy, NY: Mapinfo Corporation, 67-70.

5. Rao JNK, Scott AJ, 1981. The analysis of categorical data from complex sample surveys: chi-squared tests for goodness of fit and independence in two-way tables. J Am Stat Assoc 76: 221230.

6. StataCorp, 2003. Stata Statistical Software: Release 8.0. College Station, TX: Stata Corporation.

7. Survey Data, Reference Manual, 2003. Release 8. College Station, TX: Stata Press.

8. Snounou G, Viriyakosol S, Zhu XP, Jarra W, Pinheiro L, do Rosario VE, Thaithong S, Brown KN, 1993. High sensitivity of detection of human malaria parasites by the use of nested polymerase chain reaction. Mol Biochem Parasitol 61: 315-320.

9. Snounou G, Pinheiro L, Goncalves A, Fonseca L, Dias F, Brown KN, do Rosario VE, 1993. The importance of sensitive detection of malaria parasites in the human and insect hosts in epidemiological studies, as shown by the analysis of field samples from Guinea Bissau. Trans R Soc Trop Med Hyg 87: 649-653.

10. Mapping Malaria Risk in Africa/Atlas du Risque de la Malaria en Afrique (MARA/AMRA), 1998. Towards an Atlas of Malaria Risk in Africa. First technical report of the MARA/ARMA collaboration. Durban, South Africa. Available from http:// www.mara.org.za

11. Beier JC, Killen GF, Githure JI, 1999. Short report: entomologic inoculation rates and Plasmodium falciparum malaria prevalence in Africa. Am J Trop Med Hyg. 61: 109-113.

12. Craig M, Bredenkamp B, Vaughan Williams C, Roussow E, Kelly V, Kleinschmidt I, Martineau A, Henry G, 2002. Field and laboratory evaluation of ten rapid malaria diagnostic tests. Trans R Soc Trop Med Hyg 96: 258-265.

13. Roche J, Ayecaba S, Amela C, Alvar J, Benito A, 1996. Epidemiological characteristics of malaria in Equatorial Guinea. Res Rev Parasitol 56: 99-104.

14. Roche J, De Diego JA, Penin P, Santos M, Del Rey J, 1991. An epidemiological study of malaria in Bioko and Annobón islands (Equatorial Guinea). Ann Trop Med Parasitol 85: 477487.

15. Kleinschmidt I, Omumbo J, Briët O, van de Giesen N, Sogoba N, Mensah NK, Windmeijer P, Moussa M, Teuscher T, 2001. An empirical malaria distribution map for West Africa. Trop Med Int Health 6: 779-786.

16. Baird JK, Masbar S, Basri H, Tirtokusumo S, Subianto B, Hoffman SL, 1998. Age-dependent susceptibility to severe disease with primary exposure to Plasmodium falciparum. J Infect Dis 178: 592-595.

17. Gupta S, Snow RW, Donnelly C, Marsh K, Newbold C, 1999. Immunity to severe malaria is acquired after one or two exposures. Nat Med 5: 340-343.

18. Kleinschmidt I, Sharp B, 2001. Patterns in age-specific malaria incidence in a population exposed to low levels of malaria transmission intensity. Trop Med Int Health 6: 986-991.

19. Snow RW, Craig M, Deichmann U, Marsh K, 1999. Estimating mortality, morbidity and disability due to malaria among Africa's non-pregnant population. Bull World Health Organ 77: 617-618.

20. World Health Organization/UNICEF, 2003. The Africa Malaria Report. Geneva: World Health Organization.

21. Smith TA, Leuenberger R, Lengeler C, 2001. Child mortality and malaria transmission intensity in Africa. Trends Parasitol 17: 145-149.

22. Arudo J, Gimnig JE, ter Kuile FO, Kachur SP, Slutsker L, Kolczak MS, Hawley WA, Orago AS, Nahlen BL, PhillipsHoward PA, 2003. Comparison of government statistics and demographic surveillance to monitor mortality in children less than five years old in rural western Kenya. Am J Trop Med Hyg 68 ( 4 Suppl): 30-37.

23. Mabaso MLH, Sharp B, Lengeler C, 2004. Historical review of malarial control in southern Africa with emphasis on the use of indoor residual house-spraying. Trop Med Int Health 9: 846856.

24. Sharp B, van Wyck P, Sikasote J, Banda P, Kleinschmidt I, 2002. Malaria control by residual insecticide spraying in Chingola and Chililabombwe, Copperbelt Province, Zambia. Trop Med Int Health 7: 732-736. 